\title{
CARACTERIZAÇÃO DE NANOLÂMINAS DE GRAFENO (NG) PRODUZIDAS PELO MÉTODO DA ESFOLIAÇÃO QUÍMICA
}

\author{
A. S. XAVIER ${ }^{1}$, D. R. ALCÂNTARA ${ }^{1}$, D. F. GALVÃO ${ }^{1}$, M. N. MORAIS ${ }^{1}$, T. S. T. \\ GARCIA $^{1}$ e S. L. NOGUEIRA ${ }^{1}$ \\ ${ }^{1}$ Centro Universitário de Patos de Minas, Departamento de Engenharia Química \\ E-mail para contato: andressasqx@ @hotmail.com
}

\begin{abstract}
RESUMO - Quimicamente similar aos nanotubos de carbono, o grafite tem o potencial para ser uma excelente nanocarga. Sendo assim, modificou-se e caracterizou-se amostra de grafite comercial através de esfoliação química para produção de nanolâminas de grafeno (NG). Para a caracterização das amostras, realizou-se teste de dispersão, espectroscopia Raman e condutividade. Notou-se que a amostra de NG apresentou uma eficiente dispersão em surfactante comprovando assim a sua formação, diferente da amostra de grafite, onde os flocos precipitaram. $\mathrm{Na}$ análise de espectroscopia Raman, o grafite apresentou um pico intenso e característico em relação à sua vibração, enquanto os picos alargados e deslocados de NG indicam um aumento em intensidade, o que caracteriza a mudança na estrutura do material. Desta forma, foi possível detectar através da estrutura eletrônica do grafeno, a produção das nanolâminas. Além disso, submeteram-se as amostras ao teste de condutividade elétrica, para avaliação e confirmação do aumento de suas condutividades após a esfoliação química.
\end{abstract}

\section{INTRODUÇÃO}

Um dos avanços recentes na área da engenharia é a produção de dispositivos com dimensões cada vez menores, sem alterar suas propriedades. Quimicamente similar aos nanotubos de carbono e estruturalmente análogo às camadas de silicatos, o grafite tem o potencial para ser uma excelente nanocarga, sob a forma de camadas individuais de grafeno ou agregados de nanolâminas. O grafite natural como ocorre na natureza não pode ser utilizado como nanocarga devido à ligação química existente em suas camadas, bem como ao espaçamento entre elas, por esta razão precisa ser modificado quimicamente (GOPAKUMAR, 2004).

As nanolâminas de grafeno são compostas de carbonos constituídos por camadas com espessura atômica extraídas do grafite. Elas são consideradas um dos materiais mais finos no que diz respeito a sua estrutura, e apresentam flexibilidade, transparência, impermeabilidade e condutividade elétrica. Além disso, também apresentam um grande potencial de condução térmica e são mais duras e resistentes que diamante (CORREA, 2010).

Por estes motivos as nanolâminas de grafeno têm chamado muito a atenção da ciência devido às suas impressionantes propriedades físicas, químicas e mecânicas. Atualmente, o grafeno é obtido por processos distintos e dentre estes se destaca a sua obtenção por rotas 
químicas. Este método é considerado pela literatura o mais promissor para a produção de grafeno em larga escala (COMPTON \& NGUYEN, 2010).

Deste modo, este trabalho objetiva a obtenção e caracterização de nanolâminas de grafeno através da esfoliação química, propondo uma modificação no processo desenvolvido por SILVA e SCARPIN, 2013 visando melhor aproveitamento e menor tempo de produção.

\section{METODOLOGIA}

As nanolâminas foram obtidas no laboratório de química analítica do Centro Universitário de Patos de Minas (UNIPAM), através do seguinte procedimento:

Partiu-se de uma mistura 4:1 contendo ácido sulfúrico (PA), ácido nítrico (PA) e 20g de grafite comercial. A solução foi submetida a uma agitação mecânica seguida de 24 horas de repouso até a decantação. Nessa primeira etapa obteve-se o grafite intercalado (GI). Retirouse o excesso de sobrenadante e realizou-se a diluição da solução de GI decantada seguida de filtração a vácuo. Para sua neutralização lavou-se o que ficou retido no papel filtro com solução de $\mathrm{NaOH}(1 \mathrm{~mol} / \mathrm{L})$. Realizou-se filtração simples, transferindo o resíduo de GI para um cadinho de porcelana. $\mathrm{O}$ grafite intercalado foi aquecido a $1000^{\circ} \mathrm{C}$ durante $30 \mathrm{~s}$. Após esse tratamento térmico obtém-se o grafite expandido (GE). Com o GE obtido, fez-se uma suspensão de álcool etílico $70 \%$ submersa em um banho de ultrassom durante $8 \mathrm{~h}$. Em seguida filtrou-se a solução e repetiu-se o processo de secagem e o choque térmico a $1000^{\circ} \mathrm{C}$, resultando em NG. As etapas de transformação do grafite em NG estão esquematizadas na figura 1, a seguir:

Figura 1 - Esquema de modificação do grafite

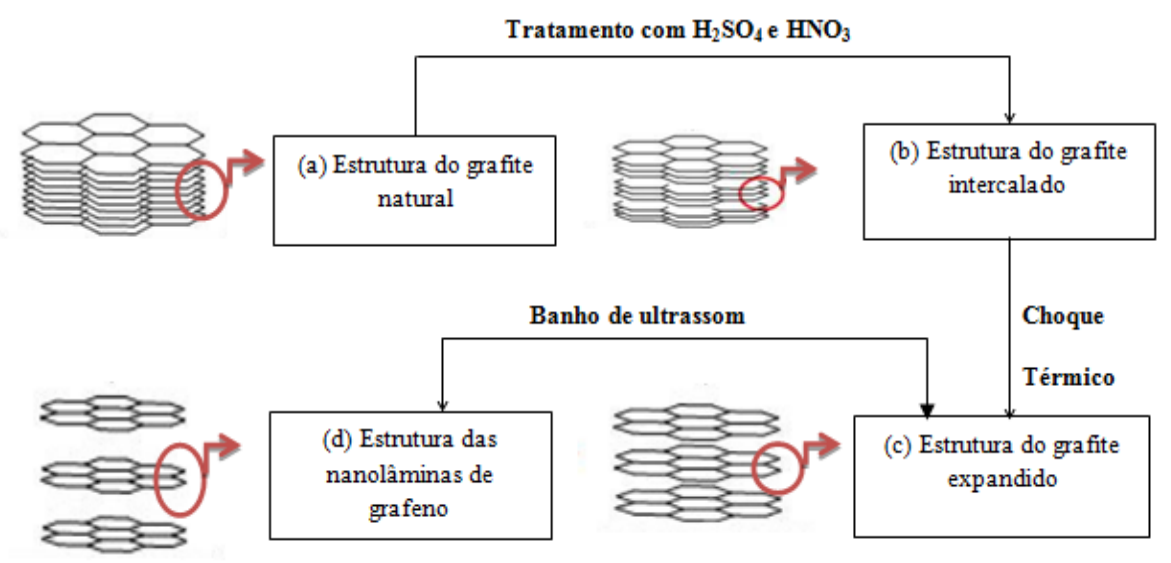

Em (a) observa-se que o grafite é composto por diversas camadas de átomos de carbono hibridizados em $\mathrm{sp}^{2}$. O tratamento químico pela adição dos ácidos concentrados acrescido de intensa agitação promove além da purificação, a oxidação do grafite, provocando o espaçamento das nanolâminas que formam a estrutura do GI (b). Quando o GI sofre um tratamento térmico drástico, os agentes intercalantes são volatilizados fragilizando assim as ligações entre os planos e fazendo com que ocorra a expansão entre as camadas, resultando no GE (c). Com as interações enfraquecidas, o GE é submetido ao banho ultrassônico para sua 
quebra definitiva. As ligações $\pi$ quebradas interagem formando com a ligação $\sigma$ uma dupla ligação entre carbonos, formando a estrutura das NG (d) (JANUARIO, 2014).

\subsection{Modificação dos agentes intercalantes}

Visando comparar as propriedades elétricas, realizaram-se outros dois testes trocando-se um dos agentes intercalantes por outros ácidos, utilizando a mesma metodologia. Substituiuse o ácido sulfúrico por ácido clorídrico e também por ácido acético.

\subsection{Caracterização das NG produzidas}

Para caracterizar as nanolâminas, analisaram-se duas amostras, uma contendo NG e outra contendo grafite natural, ambas na mesma concentração em solução aquosa de surfactante. As amostras ficaram submersas em um banho ultrassônico por 2 horas, para análise da dispersão das moléculas. Além disso, as amostras foram analisadas no espectro Raman, verificando sua modificação pelas vibrações carbonosas. Por fim, realizaram- se análises das amostras de todas as etapas de produção no condutivímetro de bancada após ultrassom de ponta, para avaliação do aumento da condutividade em cada processo.

\section{RESULTADOS E DISCUSSÕES}

O método proposto por SILVA e SCARPIN, 2013 utilizava primeira etapa do processo, bomba peristáltica para drenagem dos ácidos, seguida de um intervalo de 24 horas para a posterior filtragem da mistura de ácido nítrico, sulfúrico e grafite. $\mathrm{O}$ trabalho apresentado propõe uma diluição da mistura concentrada para facilitar a filtração e em seguida lavagem com $\mathrm{NaOH}$ para neutralização. Esta modificação no método resultou em uma economia de tempo, além de uma menor perda de GE durante o processo. Alérm disso, o produto final (NG) foi pesado para avaliação do rendimento que foi de aproximadamente $70 \%$, visto que partiram-se de $20 \mathrm{~g}$ de grafite e obtiveram-se $14 \mathrm{~g}$ de NG.

A figura 2 a seguir representa a diferença de dispersão das amostras em meio surfactante, após o banho de ultrassom. A amostra 1 que está à esquerda contém as NG e a amostra 2, grafite natural, ambas em meio surfactante à mesma concentração. Dispersão é qualquer disseminação de uma substância ao longo de todo o volume de outra substância. Assim, considera-se a disseminação de grafite e/ou NG em líquidos como dispersão. Nota-se que a amostra 1 apresentou uma eficiente dispersão, devido a sua leveza, homogeneidade e baixa densidade, comprovando assim a formação das NG. Já a amostra 2 não apresentou uma boa dispersão, visto que os flocos de grafite permaneceram aglomerados no fundo do vidro, ficando evidente a diferença entre as duas amostras.

Figura 2 - Dispersão em banho ultrassônico (NG à esquerda e grafite à direita)

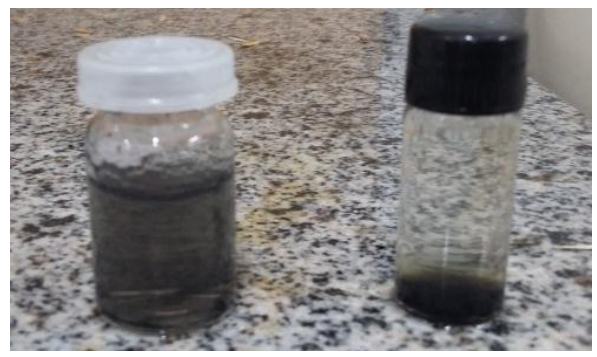


A espectroscopia RAMAN é uma técnica de caracterização estrutural e eletrônica muito utilizada nos materiais carbonosos (CHILDRES et al, 2010). As bandas originadas no espectro Raman indicam os defeitos na estrutura do material ou os tipos de vibração dos átomos no plano. Desta forma, através desta técnica torna-se possível identificar a produção de nanolâminas de grafeno - NG.

A figura 3 apresenta os espectros Raman para o grafite comercial - GR, utilizado como precursor nas esfoliações em estudo, grafite intercalado - GI (amostra resultante da etapa de tratamento com ácido), grafite expandido - GE (amostra resultante da neutralização e tratamento térmico) e das nanolâminas de grafeno - NG. Todos os espectros exibem picos característicos na região entre 1200 e $3000 \mathrm{~cm}^{-1}$ para energia de excitação do laser de argônio em $514 \mathrm{~nm}(2,41 \mathrm{eV})$. As bandas encontradas neste intervalo são conhecidas como banda D (1200 a $\left.1400 \mathrm{~cm}^{-1}\right)$, banda G (1500 a $\left.1650 \mathrm{~cm}^{-1}\right)$ e banda 2D $\left(2600\right.$ a $\left.2750 \mathrm{~cm}^{-1}\right)$.

Figura 3 - Espectro Raman para as amostras GR, GI, GE e NG

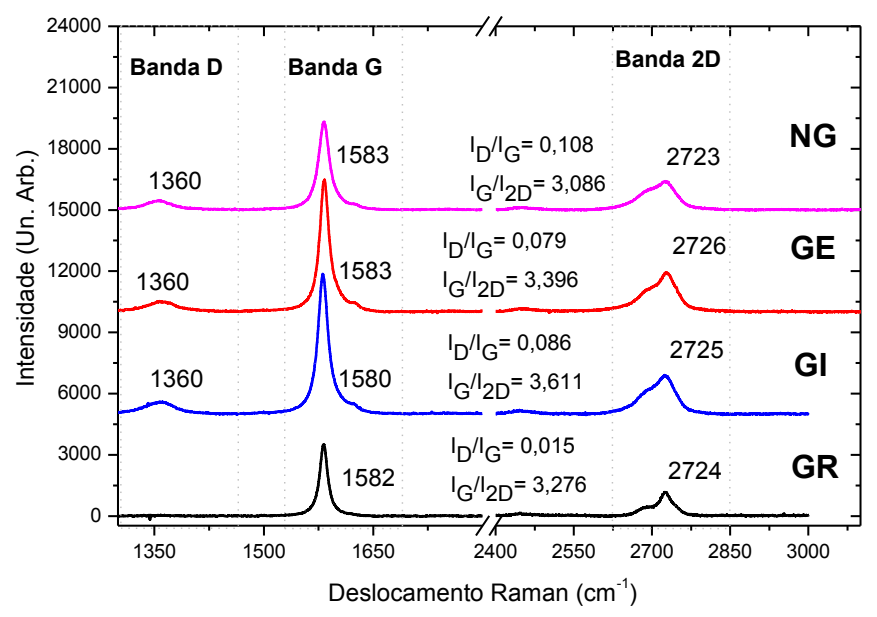

A banda $\mathrm{G}$ relaciona as vibrações $\mathrm{C}-\mathrm{C}$ do grafite, as quais estão presentes nos materiais de carbono. Por outro lado, a banda $\mathrm{D}$ tem relação com a desordem da estrutura hexagonal do grafite. Como a banda D está associada à pobre grafitização, ela é conhecida como a banda da "desordem" ou banda amorfa. Na figura 3 apresentada nota-se que o sinal da banda D é mais intenso referente ao grafite tratado, GI e GE e também das NGs indicando materiais estruturalmente mais desorganizados. Isso se deve ao processo de esfoliação química do grafite que introduz defeitos em sua estrutura cristalina em razão da quebra grafítica e a incorporação de grupos oxigenados pela oxidação do grafite no tratamento com ácido forte.

Uma maneira de quantificar esta desordem é a partir da razão das intensidades das bandas $\mathrm{D}$ e $\mathrm{G}$ (valor $\mathrm{I}_{\mathrm{D}} / \mathrm{I}_{\mathrm{G}}$ ). De acordo com a literatura, (FERRARI et al, 2012), o valor de $\mathrm{I}_{\mathrm{D}} / \mathrm{I}_{\mathrm{G}}$ próximo de zero é um indicativo de um material grafítico cristalino, porém quando esse valor aumenta, o material grafítico perde sua cristalinidade e torna-se estruturalmente mais desorganizado. Baseado nesses valores, que também estão apresentados na figura $3, \mathrm{I}_{\mathrm{D}} / \mathrm{I}_{\mathrm{G}}=$ 0.015 confirma-se o elevado grau de cristalinidade do grafite. Entretanto, os valores de $I_{D} / I_{G}$ bem próximos para GI, GE e NG revelam a presença de uma maior quantidade de defeitos 
estruturais. Como os valores são próximos é possível inferir que ambos possuem relativamente a mesma quantidade de defeitos estruturais.

A segunda ordem da banda D, ou seja, a banda 2D, é uma das mais importantes do espectro Raman, pois é a partir dela que torna-se possível a identificação de nanolâminas de grafeno no material e sobretudo, a quantidade de nanolâminas (FERRARI et al, 2006 e 2012). Quando o número de camadas de grafeno aumenta, o número de interações entre as camadas é modificado e o espectro Raman mudará de acordo com o número de modos que podem ser combinados. Desta forma, a banda 2D pode ser alargada e intensificada e a banda G também pode ser deslocada para o vermelho. Assim, o número de camadas pode ser obtido a partir da razão das intensidades da banda $2 \mathrm{D}$ e da banda $\mathrm{G}$ (valor $\mathrm{I}_{\mathrm{G}} / \mathrm{I}_{2 \mathrm{D}}$ ). Na figura nota-se que $\mathrm{o}$ espectro que apresentou menor valor para a razão $\mathrm{I}_{\mathrm{G}} / \mathrm{I}_{2 \mathrm{D}}$ foi a amostra de NG. Para quantificar o número de lâminas é preciso fazer uma deconvolução da curva para estabelecer quantitativamente este parâmetro. A partir desses resultados, é possível concluir que houve a formação de nanolâminas a partir da esfoliação de grafite, pois os espectros das amostras analisadas apresentam características estruturais diferentes de um material grafítico natural.

Para complementar a caracterização, realizou-se o teste de condutividade elétrica das amostras após dispersão em ultrassom de ponta. A tabela 1 mostra o resultado de três testes realizados modificando-se os agentes intercalantes para avaliar a alteração das propriedades elétricas das amostras, onde (A1-GI) e (A1-NG) correspondem, respectivamente, às amostras de grafite intercalado e nanolâminas de grafeno obtidas do teste com ácido sulfúrico e ácido nítrico, (B1-GI) e (B1-NG) correspondem às amostras de grafite intercalado e nanolâminas de grafeno obtidas do teste com ácido clorídrico e ácido nítrico e (C1-GI) e (C1-NG) correspondem às amostras de grafite intercalado e nanolâminas de grafeno obtidas do teste com ácido acético e ácido nítrico.

Tabela 1 - Condutividade elétrica das amostras após ultrassom de ponta

\begin{tabular}{|c|c|c|}
\hline Amostras & $\begin{array}{c}\text { Condutividade } \\
(\mathrm{mS} / \mathrm{cm})\end{array}$ & Temperatura $\left({ }^{\circ} \mathrm{C}\right)$ \\
\hline \hline Grafite comercial & 10,73 & 22,7 \\
\hline A1-GI & 11,20 & 23,5 \\
\hline A1-NG & 16,40 & 23,6 \\
\hline B1-GI & 11,98 & 22,9 \\
\hline B1-NG & 12,48 & 23,4 \\
\hline C1-GI & 10,88 & 23,6 \\
\hline CI-NG & 11,15 & 23,3 \\
\hline
\end{tabular}

No teste A1 nota-se um aumento de $4,2 \mathrm{mS} / \mathrm{cm}$ na condutividade do grafite intercalado GI para o produto final NG. No teste B1, o aumento de condutividade foi menor, $0,50 \mathrm{mS} / \mathrm{cm}$, de GI para NG. No teste C1, observa-se o menor aumento de condutividade, $0,27 \mathrm{mS} / \mathrm{cm}$ de GI para NG dentre todos os teste feitos. Comparando os resultados das amostras com o material de partida (grafite comercial), observa-se que houve aumento de condutividade em todos os testes. Com estes resultados é possível afirmar que a substituição de ácidos na intercalação acarreta uma alteração nas propriedades elétricas das NG produzidas, obtendo maiores valores nos testes feitos com ácidos mais fortes como agentes intercalantes. 


\section{CONCLUSÕES}

As mudanças da metodologia de obtenção de NG não afetaram a produção, e sim proporcionaram uma melhoria no processo, com uma maior economia de água e otimização no tempo de produção.

O objetivo de produzir NG foi confirmado pela boa dispersão das moléculas das nanolâminas em meio surfactante, comparadas ao grafite natural. Além disso, as amostras de NG foram caracterizadas por Espectroscopia Raman, sendo possível detectar através da estrutura eletrônica do grafeno, a dependência com o número de camadas das nanolâminas produzidas, reafirmando sua obtenção eficaz.

Pela análise de condutividade das amostras de todos os testes feitos, conclui-se que os ácidos fortes são melhores intercalantes e conferem às NG maior valor de condutividade elétrica.

Partindo de $20 \mathrm{~g}$ de grafite obteve-se $14 \mathrm{~g}$ de NG, ou seja, um rendimento de $70 \%$ de NG. Com isso, pode-se concluir que a esfoliação química do grafite mostra-se como uma possível rota para a preparação de nanolâminas de grafeno em maior escala, permitindo estudo em diversas aplicações, aproveitando-se de suas propriedades impressionantes.

\section{REFERENCIAS}

CHILDRES, I.; JAUREGUIB, L. A.; PARKB, W.; CAOAB, H.; CHEN, Y. P. Raman spectroscopy of graphene and related materials. Chapter 19 (2010).

COMPTON, O. C.; NGUYEN, S. T. Graphene oxide, highly reduced graphene oxide, and graphene: versatile building blocks for carbon-based materials.(2010), 711-723.

CORREA D. R. N. A síntese do Grafeno: O premio Nobel de Física de 2010 - 2010.

FERRARI, A. C.; MEYER, J. C.; SCARDACI, V.; CASIRAGHI, C.; LAZZERI, M.;MAURI, F.; PISCANEC, S.; JIANG, D.; NOVOSELOV, K. S.; ROTH, S. and GEIM, A. K. Raman Spectrum of Graphene and Graphene Layers. PRL, 2006.

FERRARI, A. C.; MEYER, J. C.; SCARDACI, V.; CASIRAGHI, C.; LAZZERI, M.;MAURI, F.; PISCANEC, S.; JIANG, D.; NOVOSELOV, K. S.; ROTH, S. and FIM, A C. Síntese e Propriedades de Nanocompósitos de Polietileno/Nanolâminas de Grafeno Obtidos Através de Polimerização In Situ. 2012. 90p. Tese (Doutorado) - Faculdade de Ciência dos Materiais, Universidade Federal do Rio Grande do Sul, Porto Alegre, 2012.

GOPAKUMAR, T. G.; PEGÉ, D. J. Y. S. Polym. Eng. Sci, 2004, 44, 1162.

JANUARIO, A. C.; REMÉDIO, B. R.; SOUZA, R. A.; 'Produção de grafeno pelo método da esfoliação química e suas potencias aplicações", p. 13392-13398. In: Anais do XX Congresso Brasileiro de Engenharia Química - COBEQ 2014[BlucherChemicalEngineeringProceedings, v. 1, n. 2]. São Paulo: Blucher.

SILVA, C. R. e SCAPIN, K. Síntese do Grafeno e Produção de Nanocompósitos Poliméricos. Trabalho de conclusão de curso - Centro Universitário das Faculdades Associadas de Ensino - UNIFAE, 2013. 